\title{
CLIC4 mediates TGF-ß1-induced fibroblast-to-myofibroblast transdifferentiation in ovarian cancer
}

\author{
QIN YAO ${ }^{1,3}, \mathrm{XUN}^{2}$ QU ${ }^{2}$ QIFENG YANG ${ }^{2}$, MINGQIAN WEI $^{3}$ and BEIHUA KONG ${ }^{1}$ \\ ${ }^{1}$ Department of Obstetrics and Gynaecology, ${ }^{2}$ Institute of Basic Medical Sciences, Qilu Hospital, Shandong University, \\ Ji'nan 250012, Shandong, P.R. China; ${ }^{3}$ Division of Molecular Medicine and Gene Therapy, Griffith Institute for Health and \\ Medical Research, School of Medical Science, Griffith University, Gold Coast Campus, Southport, Qld 4222, Australia
}

Received April 15, 2009; Accepted May 18, 2009

DOI: $10.3892 /$ or_00000469

\begin{abstract}
Stromal myofibroblasts, activated by crosstalk signaling between the tumour and stroma, play a critical role in tumour development and progression. Chloride intracellular channel 4 (CLIC4) may be functionally import for tumour stromal fibroblast-to-myofibroblast transdifferentiaton, but the molecular mechanism of the process has not been addressed. In this study, the expression of CLIC4 in ovarian cancer tissues was analyzed by immunohistochemistry, and we used an indirect co-culture model of ovarian cancer cells and normal fibroblasts to demonstrate the molecular pathway in which CLIC4 participated during the fibroblast-tomyofibroblast transdifferentiation. The results showed that the expression of CLIC4 in $96.7 \%$ of ovarian cancer stroma and correlated with the up-regulation of myofibroblast marker $\alpha$-SMA. Conditioned medium from ovarian cancer cells (CM) or transforming growth factor- $\beta 1$ (TGF- $\$ 1$ ) increased cellular reactive oxygen species (ROS) levels in fibroblasts, which initiated up-regulation of CLIC4 expression, then resulted in myofibroblast conversion. Moreover, inhibition of
\end{abstract}

Correspondence to: Professor Beihua Kong, Department of Obstetrics and Gynaecology, Qilu Hospital, Shandong University, 107 Wenhuaxi Road, Ji'nan 250012, Shandong, P.R. China E-mail: kongbeihua@yahoo.com.cn

Professor Mingqian Wei, Division of Molecular Medicine and Gene Therapy, Griffith Institute for Health and Medical Research, School of Medical Science, Griffith University, Gold Coast Campus, Southport, Qld 4222, Australia

E-mail: m.wei@griffith.edu.au

Abbreviations: CLIC4, chloride intracellular channel 4; $\mathrm{CM}^{\mathrm{SKOV} 3}$, conditioned medium from SKOV3; DCF-DA, 2'-7'-dihydrodichlorofluorescein diacetate; GST, glutathione S-transferase; NAC, N-acetyl-L-cysteine; ROS, reactive oxygen species; siRNA, small interfering RNA; TGF- $\beta 1$, transforming growth factor- $\beta 1$

Key words: reactive oxygen species, chloride intracellular channel 4, transforming growth factor- $\beta 1$, tumour and stroma interaction, myofibroblasts
CLIC4 significantly reduced the expressions of factors related to the phenotype and functions of myofibroblasts, such as $\alpha$ SMA, VEGF and HGF. These results suggest that ROSinitiated CLIC4 up-regulation is required for TGF- $\$ 1$-induced fibroblast-to-myofibroblast transdifferentiaton in ovarian cancer, indicating that inhibiting the CLIC4 might have therapeutic potential targeting tumour stroma.

\section{Introduction}

Epithelial ovarian cancer is the most lethal disease among gynaecologic malignancies (1-3). For years, it has been dubbed as a 'silent killer' - causing no symptom till it is too advanced at the time of diagnosis (2). Five-year survival for patients with advanced ovarian cancer improved little even with the treatment of optimal cytoreductive surgery and systemic combination chemotherapy (3). Increasing evidence has indicated that cancer development is facilitated by interaction between tumour cells and activated stromal cells (4). The tumour stroma (also referred to as 'reactive stroma') is characterized by marked alterations in the phenotype and expression profile of fibroblast-like cells. These cells commonly express $\alpha$-SMA and thus are termed as myofibroblasts $(5,6)$. Stromal fibroblasts are located at the tumour border near the invasion front, and play a crucial role in tumour progression (7). Recent studies demonstrated that coculturing of fibroblasts with tumour cells can promote tumour invasion and angiogenesis $(8,9)$.

Myofibroblasts are recruited from different sources during cancer development and the invasion progression $(10,11)$. Myofibroblasts can differentiate from the local fibroblast population in the epithelial stroma on transforming growth factor- $\beta 1$ (TGF- 1 ) secreted by tumour cells stimulation. Tumour-associated myofibroblasts also transdifferentiate from non-malignant or epithelial derived carcinoma cells via epithelial-mesenchymal transition (11). Moreover, myofibroblasts are recruited or derived from distant fibroblasts and bone marrow progenitor cells $(12,13)$. Although it has been shown that the conversion from fibroblasts to myofibroblasts constitutes the major source of myofibroblasts in tumour stroma, the molecular mechanism underlying fibroblast-tomyofibroblast transdifferentiation is still not fully understood.

Chloride intracellular channel 4 (CLIC4), a chloride channel of intracellular organelles, regulates intracellular $\mathrm{pH}$ 
and cell volume. Besides its presence on the organelle membrane, CLIC4 exists in soluble form in the cytoplasm and nucleus acting as signalling protein or channel regulator (14). Transcription level of CLIC4 is up-regulated when fibroblasts transdifferentiate into myofibroblasts induced by TGF- 31 , and more importantly CLIC4 is highly expressed in myofibroblasts of breast cancer (15). The work of Littler and colleagues (16) shows that functional activity of CLIC4 depends on its redox state and oxidative conditions enhance membrane binding and channel activity of CLIC4. Recent findings also reveal that reactive oxygen species (ROS) can alter gene expression level associated with cell differentiation, including fibroblast-to-myofibroblast transdifferentiation and epithelialmesenchymal transition $(10,17,18)$. Hence, we hypothesized that the factors involved in fibroblast-to-myofibroblast transdifferentiation, such as tumour-cell-derived TGF- 31 , promoted the generation of intercellular ROS, which in turn initiated up-regulation of CLIC4 expression, thus triggering fibroblastto-myofibroblast conversion. Agents that block CLIC4 might inhibit such transdifferentiation and delay myofibroblastdependent tumour progression.

To test the hypothesis, we designed this study to confirm whether CLIC4 was highly expressed in epithelial ovarian cancer tissues and to examine the pathway in which CLIC4 participated during the fibroblast-to-myofibroblast transition induced by TGF- $\$ 1$ from ovarian cancer cells.

\section{Materials and methods}

Tissue specimens. Tissue specimens were collected from patients who underwent surgery at the Department of Obstetrics and Gynaecology, Qilu Hospital, Shandong University, following informed consent and approval by the local Human Research Ethics Committee. The specimens consisted of 30 primary epithelial ovarian cancer and 8 normal ovarian tissues. Of 30 patients, 23 were serous carcinomas, 5 mucinous carcinomas and 2 endometrioid carcinomas. Three cases were in stage I, 2 in stage II and 25 in stage III. For paraffin-embedded sections, surgical tissues were fixed in $10 \%$ formalin and then embedded in paraffin. For tissue culture, ovaries were placed in DMEM (Gibco) and immediately transported on ice to the laboratory.

Cell culture. Both human epithelial ovarian cancer cell line SKOV3 and human fetal lung fibroblast cell line MRC-5 were obtained from Basic Medicine Research Institute, Qilu Hospital, Shandong University, P.R. China. Human ovarian primary fibroblasts were isolated as described previously (19). These cells were cultured in DMEM containing 10\% heat-inactivated fetal bovine serum (Gibco) and 100 units $/ \mathrm{ml}$ penicillin/streptomycin. The cultures were maintained at $37^{\circ} \mathrm{C}$ in an atmosphere of $5 \% \mathrm{CO}_{2}$. The purity of primary stromal cells was $>98 \%$ with keratin and vimentin staining (20). Ovarian fibroblasts between passage 3 and 8 were used for all experiments.

Treatment of cells with TGF- $\beta 1$, conditioned medium from SKOV3 (CM $\left.\mathrm{CKOV}^{\mathrm{SK}}\right), \mathrm{N}$-acetyl-L-cysteine (NAC) or IAA-94. After SKOV3 cells were cultured in serum-free medium for $48 \mathrm{~h}$, the conditioned medium was collected, clarified by centrifugation and stored at $-20^{\circ} \mathrm{C}$ for future use.
When MRC-5 cells and human ovarian primary fibroblasts reached subconfluence, the medium was replaced by serum-free medium, serum-free medium with $10 \mathrm{ng} / \mathrm{ml}$ human recombinant TGF-ß1 (PeproTech EC Ltd., UK) or CM ${ }^{\text {SKOv3 }}$, then cultured for another $48 \mathrm{~h}$. In order to study the inhibitory functions of NAC and IAA-94, subconfluent fibroblasts were incubated with $5 \mathrm{mM}$ NAC for $4 \mathrm{~h}$ or $10 \mu \mathrm{M}$ IAA-94 for $24 \mathrm{~h}$ before addition of TGF- $\beta 1$ or $\mathrm{CM}^{\text {SKOv3 }}$.

Immunochemical and immunofluorescent staining. For immunohistochemical staining, the sections were deparaffinized, rehydrated followed by antigen retrieval. The cells were fixed with ice-cold acetone for immunocytochemical analysis. Afterwards, these sections and cells were blocked with $10 \%$ normal goat serum, then incubated with rabbit antiCLIC4 polyclonal antibody (Lifespan Biosciences, USA) and rabbit anti- $\alpha$-SMA monoclonal antibody (Abcam, UK) at $4{ }^{\circ} \mathrm{C}$ overnight, respectively. After washing with PBS, the slides were incubated with anti-rabbit IgG secondary antibody for $45 \mathrm{~min}$ and biotinylated horseradish peroxidase solution for $45 \mathrm{~min}$. Finally, the slides were stained with diaminobenzidine and observed under light microscope. For immunofluorescent staining, cells were stained with FITC conjugated anti-rabbit IgG secondary antibody, nuclei with 4,6-diamidino-2phenylindole (DAPI). Images were collected on a Zeiss laserscanning confocal microscope.

Western blot analysis. Cells were lysed in RIPA lysis buffer (50 mM Tris- $\mathrm{HCl} \mathrm{pH} 7.6,150 \mathrm{mM}$ sodium chloride, $1.0 \%$ NP-40, $1 \%$ sodium deoxychlorate, and $0.1 \%$ SDS) plus protease inhibitors. Cell nuclear and cytoplasmic proteins were extracted using Nuclear/Cytosol Extraction Kit (Pierce Biotechnology, USA). The expressions of CLIC4 and $\alpha$-SMA were characterized by Western blots using polyclonal antiCLIC4 antibody and monoclonal anti- $\alpha$-SMA antibody as primary antibodies. Reaction products of $\alpha$-tubulin were used to control for total or cytoplasmic protein loading, Lamin $\mathrm{A}+\mathrm{C}$ for normalizing nucleic protein loading. The band intensities were measured by densitometry using the Quantity One software (Bio-Rad, CA, USA).

Analysis of intracellular ROS. The intracellular ROS generation was assayed using 2'-7'-dichlorofluorescein diacetate (DCF-DA) (Sigma) dye as previously described (10). DCF-DA can diffuse into cells readily, where it is hydrolyzed to the non-fluorescent polar derivative DCF-DA and trapped within cells. In the presence of the oxidant, DCF-DA turns to highly fluorescent 2'-7'-dichlorofluorescein (DCF).

Small interfering RNA (siRNA) transfection. The siRNAs used in this study were designed by and purchased from Qiagen. The sequences of siRNA were as follows: CLIC4 validated siRNA, 5'-GCAGUACAAUGAUUAGUAAdTdT-3' (sence) and 5'-UUACUAAUCAUUGUACUGCdTdA-3' (antisence); negative control siRNA, 5'-UUCUCCGAACGUGUC ACGUdTdT-3' (sence) and 5'-ACGUGACACGUUCGG AGAAdTdT-3' (antisence); MAPK1 positive control siRNA, 5'-UGCUGACUCCAAAGCUCUGdTdT-3' (sence) and 5'CAGAGCUUUGGAGUCAGCAdTdT-3' (antisence). Lipofectamine 2000 reagent was used with a final concentration of $30 \mathrm{nM}$ of each siRNA. After $16 \mathrm{~h}$ of transfection, 
A

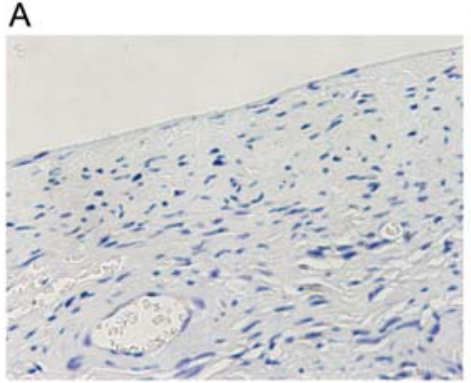

B

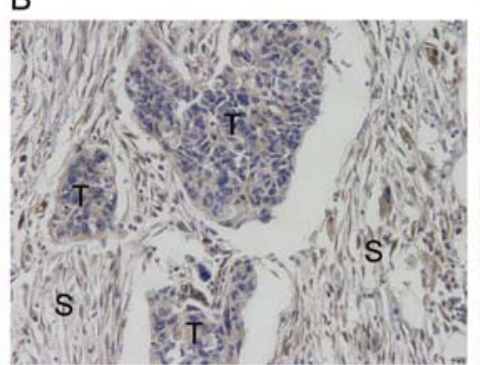

C

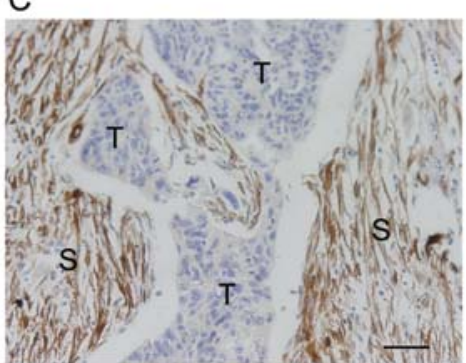

Figure 1. Immunohistochemical stainings of CLIC4 and $\alpha$-SMA in normal ovarian tissue and cancer tissue. (A) Normal ovarian tissue was negative for CLIC4 expression. (B) CLIC4 expression was detected in the stroma and epithelium of tumour. (C) $\alpha$-SMA expression is shown in the same tumour stroma. S indicates tumour stroma, T indicates tumour epithelium. Scale bar, $50 \mu \mathrm{m}$.

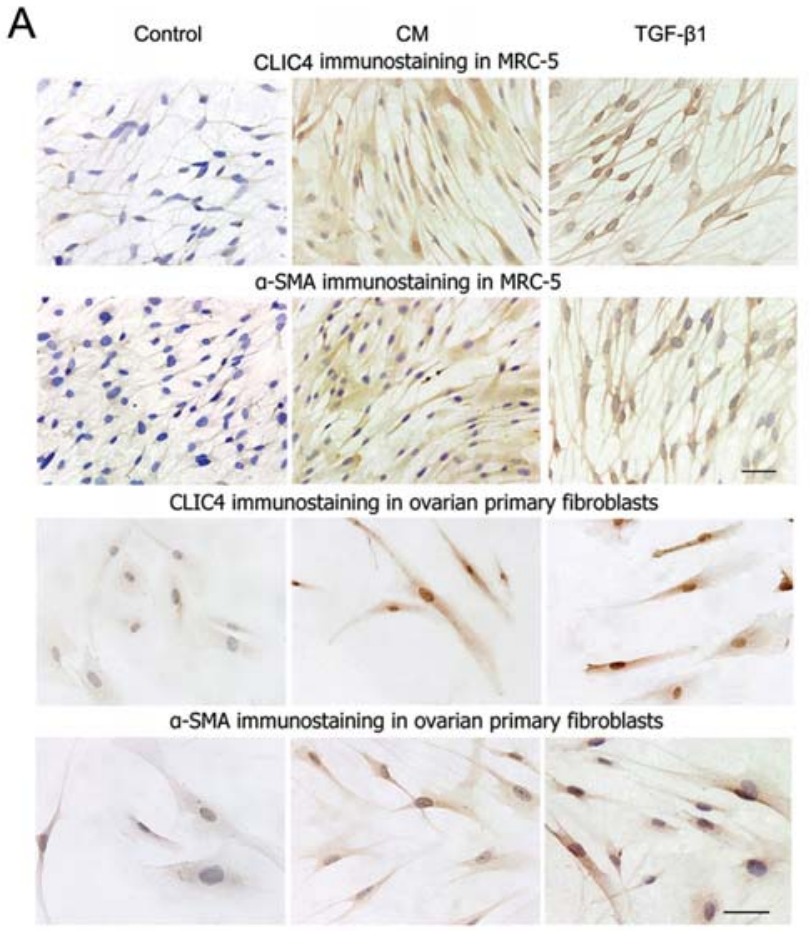

B

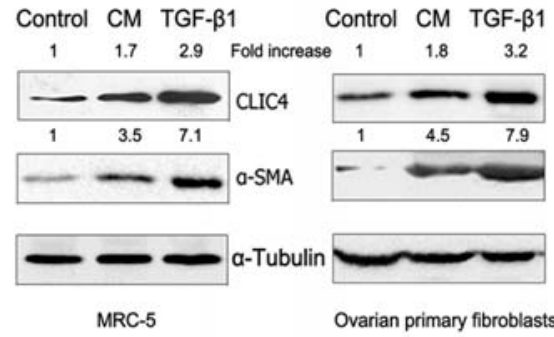

C

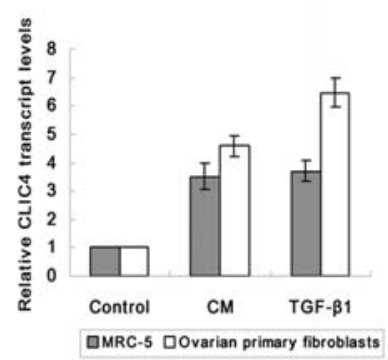

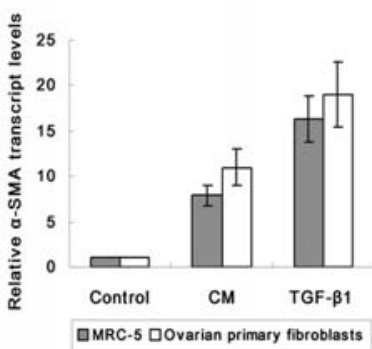

Figure 2. Expression levels of CLIC4 and $\alpha$-SMA in fibroblasts up-regulate during transdifferiation of fibroblasts to myofibroblasts. Subconfluent MRC-5 cells or primary ovarian fibroblasts were cultured in serum-free medium (control), in $\mathrm{CM}^{\mathrm{SKOV} 3}$ or treated with $10 \mathrm{ng} / \mathrm{ml} \mathrm{TGF}-\beta 1 \mathrm{for} 48 \mathrm{~h}$. CLIC4 and $\alpha-\mathrm{SMA}$ expressions were observed using immunocytochemistry (A), Western blotting (B) and real-time RT-PCR (C). Scale bar, $50 \mu \mathrm{m}$.

cells were incubated in serum-free medium with or without $10 \mathrm{ng} / \mathrm{ml}$ TGF-ß1 or $\mathrm{CM}^{\text {SKOV3 }}$ for another $48 \mathrm{~h}$.

Real-time PCR. Total RNA was extracted from cells using TRIzol reagent (Invitrogen). RNA was reverse transcribed with oligo(dT) and M-MLV reverse transcriptase (Promega). The primers were designed as follows: $\alpha$-SMA (accession no. NM_001613), 5'-AGGTAACGAGTCAGAGCTTTGGC-3' (sence) and 5'-CTCTCTGTCCACCTTCCAGCAG-3' (antisence); CLIC4 (accession no. NM_013943), 5'-CACGTAAA TTTCTGGATGGCAATG-3' (sence) and 5'-ATCACTGGG ACAGGTATTGGTGAAC-3' (antisence); VEGF (accession no. NM_001025366), 5'-CCTGGTGGACATCTTCCAGGA GTACC-3' (sence) and 5'-GAAGCTCATCTCTCCTATGTG CTGGC-3' (antisence); HGF (accession no. NM_000601), 5'-GTAAATGGGATTCCAACACGAACAA-3' (sence) and 5'-TGTCGTGCAGTAACAACCAACTC-3' (antisence); B-actin (accession no. NM_001101), 5'-AACTCCATCATG
AAGTGTGA-3' (sence) and 5'-ACTCCTGCTTGCTGATC CAC-3' (antisence). B-actin was chosen as house-keeping gene. The quantification was performed with SYBR Green (Takara Biotechnology, Inc., Japan). Samples were analysed using LightCycler ${ }^{\circledR} 2.0$ Instrument (Roche Applied Science, Switzerland).

Statistical analysis. The data represent means \pm SD of three independent experiments. Statistical differences were analysed with the Student's t-test. Differences were considered significant at $\mathrm{P}$-value $<0.05$.

\section{Results}

The expression of CLIC4 is up-regulated in epithelial ovarian cancer tissues. CLIC4 may play an important functional role in stromal myofibroblasts, which are critical in tumour development and progression. As shown in Fig. 1B, CLIC4 
was detected in epithelium and stroma of patients with primary epithelial ovarian tumours. Positive CLIC4 staining in stroma was shown in $96.7 \%$ (29/30) of patients. Moreover, the expression of CLIC4 in cancer stroma was correlated with up-regulation of a well-accepted myofibroblast marker, $\alpha$-SMA (Fig. 1C), which was detected in $93.3 \%$ (28/30) of the same stroma. Co-expression of CLIC 4 and $\alpha$-SMA were seen in $93.3 \%(28 / 30)$ of cases, while both negative in 1 case and CLIC4(+)/ $\alpha$-SMA(-) expression in only one. In normal tissues, previous studies have reported that CLIC4 is detected in multiple epithelial cells, such as epidermis, esophagus, colon, and vascular endothelial cells (21-23). CLIC4 expression level in the stroma is very low (21). However, ovarian surface epithelium is in fact a single layer of mesothelial cells covering the ovarian surface and contiguous with coelomic mesothelium. Our result showed (Fig. 1A) that CLIC4 was not observed in normal ovarian surface epithelium and stroma. In addition, CLIC4 staining in primary tumours had no significant correlation with the clinicopathologic features, including tumour grade, histological type and clinical stage (data not shown).

The expression of CLIC4 is up-regulated during fibroblastto-myofibroblast transdifferentiation in vitro. TGF- 31 is the major mediator in the fibroblast-to-myofibroblast conversion process. Tumour cells release large amounts of active TGF- $\$ 1$ (24). In order to define the alteration of CLIC4 expression in myofibroblast transdifferentiation, we used a myofibroblast conversion cell model where ovarian primary fibroblasts or human fibroblast MRC-5 cells were cultured with $\mathrm{CM}^{\text {SKOv3 }}$ or TGF-31. Fig. 2A showed that the expression of $\alpha$-SMA in the cytoplasm of the activated fibroblasts increased expectedly in the presence of $\mathrm{CM}^{\mathrm{SKOV} 3}$ or $10 \mathrm{ng} / \mathrm{ml} \mathrm{TGF}-\beta 1$ for $48 \mathrm{~h}$ compared with that in serum-free medium control. Coincidently with up-regulation of $\alpha$-SMA expression, the activated fibroblasts displayed evident increase of CLIC4 expression both in the cytoplasm and nucleus. Furthermore, the immunoblot and real-time RT-PCR data revealed that the relative amounts of $\alpha$-SMA and CLIC4 expression in $\mathrm{CM}^{\text {SKOv3 }}$ - or TGF- 31 -treated fibroblasts were up-regulated both at the transcription and protein levels compared with the untreated control (Fig. 2B, C).

ROS mediate up-regulation of CLIC4 expression during myofibroblast transdifferentiation. ROS act as signalling molecules of TGF- $\beta 1$ in the regulation of myofibroblast differentiation $(10,17)$. Therefore, we attempted to investigate whether ROS regulated expression of CLIC 4 and $\alpha-S M A$ during fibroblast activation. We examined the intracellular ROS levels in fibroblasts using DCF-DA dye. MRC-5 cells treated with $10 \mathrm{ng} / \mathrm{ml}$ TGF- 31 or $\mathrm{CM}^{\text {SKOV } 3}$ for $1 \mathrm{~h}$ showed a significant increase in the fluorescence intensity, suggesting increased intracellular ROS (Fig. 3A). When cells were pretreated with the antioxidant NAC, the up-regulation of $\alpha$-SMA and CLIC4 expression stimulated by TGF- 31 or $\mathrm{CM}^{\text {SKOv3 }}$ was suppressed (Fig. 3B, C).

CLIC4 subcellular location is critical in its physiological functions (25). Therefore, we further assessed the expression of CLIC4 in the nucleus and cytoplasm during myofibroblast differentiation using Western blotting. As shown in Fig. 3D, the expression of CLIC4 increased not only in the cytoplasm but also in the nucleus, particularly in the nucleus when fibroblasts were treated with TGF- 11 or $\mathrm{CM}^{\text {SKov3 }}$. Pre-treatment with antioxidant NAC repressed both cytoplasmic and nuclear CLIC4 expressions.

CLIC4 is required for TGF- $\beta 1$ induced myofibroblast transdifferentiation. The above data suggested CLIC4 was implicated in the process of myofibroblast transdifferentiation. However, it is not clear whether CLIC4 serves as a late differentiation marker like $\alpha$-SMA or promotes myofibroblast conversion as an early differentiation marker. We thus reduced CLIC4 level by transfection with validated specific CLIC4 siRNA in MRC-5 fibroblasts. CLIC4 siRNA efficiently downregulated CLIC4 transcript levels by an average of $90 \%$ (Fig. 4A). The induction of $\alpha$-SMA transcription induced by TGF- 31 or $\mathrm{CM}^{\mathrm{SKOV} 3}$ was significantly blocked in CLIC4 siRNA-transfected fibroblasts (Fig. 4B). At the protein level, immunoblot and immunofluorescent data showed that silencing of CLIC4 prevented the expression of $\alpha$-SMA (Fig. 4C, D).

Blocking CLIC4 inhibits the expression of myofibroblastmediated up-regulation of pro-angiogenic factors. Angiogenesis plays a key role in tumour development by supplying the tumour with oxygen and nutrients. Recent data show that myofibroblast is the major cell type expressing proangiogenic factors in cancer stroma. Meanwhile, tumour stromal fibroblasts secret abundant angiogenic factor VEGF only after activated by tumour cells (12). Since CLIC4 is required for TGF- $\beta 1$ induced myofibroblast transdifferentiation, we next tested whether blockade of CLIC4 could prevent the generation of pro-angiogenesis factors. As expected and shown in Fig. 5, when fibroblasts were stimulated by $\mathrm{CM}^{\mathrm{SKOV} 3}$ or TGF- 31 , the expressions of VEGF and HGF increased significantly compared with that in the unstimulated fibroblasts. Whereas, pre-treatment with CLIC4 siRNA to silence CLIC4 expression or chloride channel inhibitor IAA-94 to block function of CLIC4 lowered VEGF and HGF expression levels in the activated fibroblasts by $29-80 \%$. This suggested that inhibiting CLIC4 could reduce the proangiogenesis production related to prevention of myofibroblast conversion. Furthermore, after fibroblasts were relieved from these treatments, $\mathrm{CM}^{\mathrm{SKOV} 3}$ or TGF- $\beta 1$ still could induce expressions of the factors related to the phenotype and functions of myofibrobalsts (data not shown), indicating these repressing activities were not due to cytotoxic effect.

\section{Discussion}

In the present study, we showed CLIC4 was highly expressed in ovarian cancer stroma and correlated with up-regulation of a well-accepted myofibroblast marker, $\alpha$-SMA. In vitro, the expression of CLIC4 was up-regulated during fibroblast to myofibroblast conversion induced by TGF- $\beta 1$ or $\mathrm{CM}$ from ovarian cancer cells. In addition, we showed for the first time that TGF- $B 1$ or CM stimulated the production of cellular ROS, which caused up-regulation of CLIC4, then in turn led to myofibroblast transdifferentiation. Moreover, the agents which inhibit CLIC4 alleviated the expression of 

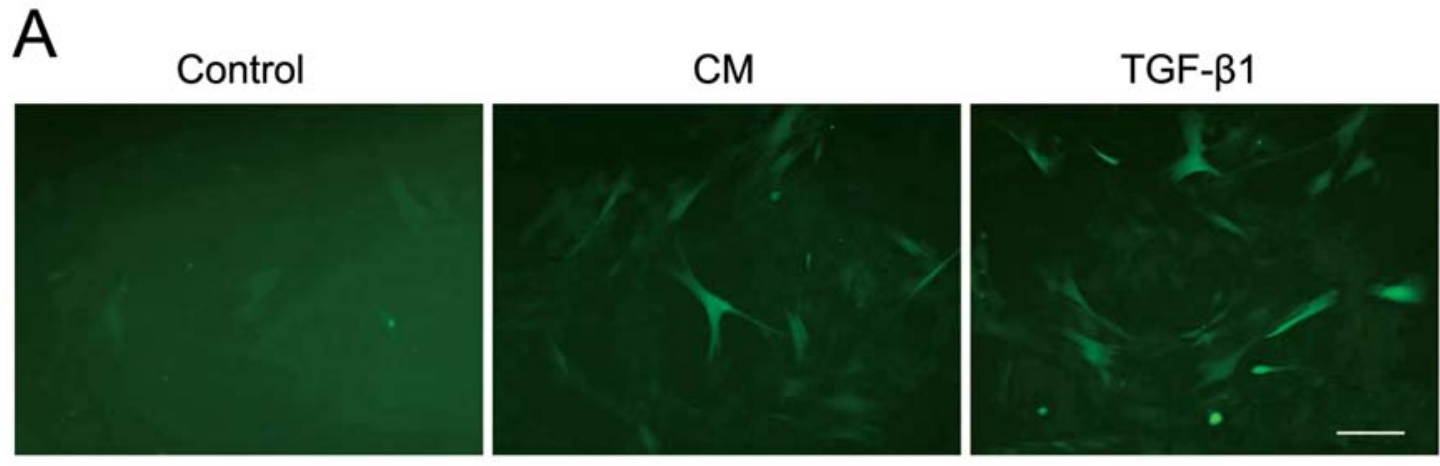

B

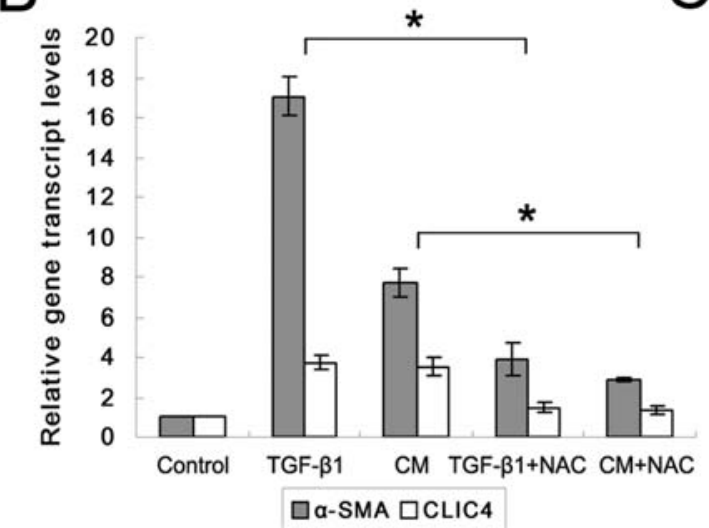

Nucleus

\section{Cytoplasm}

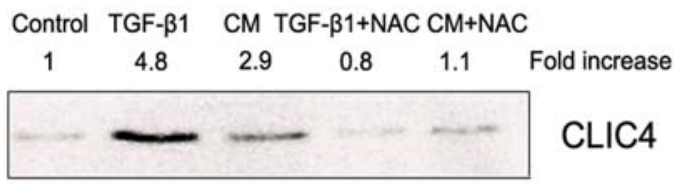

Control TGF- $\beta 1$ CM TGF- $\beta 1+N A C$ CM+NAC


Lamin A+C

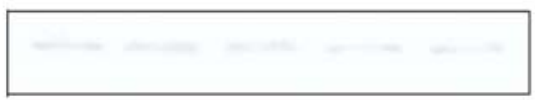

a-tubulin

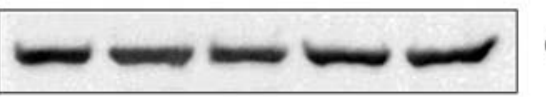

a-tubulin

Figure 3. ROS mediate up-regulation of CLIC4 expression during myofibroblast transdifferentiation. (A) CM ${ }^{\text {SKOv3 }}$ and TGF- 31 induced oxidative stress in fibroblasts. MRC-5 cells were loaded with DCF-DA, and then treated with CM ${ }^{\text {SKOv3 }}$ or 10 ng/ml TGF- 11 for another hour, cells were viewed using fluorescent microscope. Scale bar, $50 \mu \mathrm{m}$. (B and C) Myofibroblast transdifferentiation was dependent on ROS. The $\alpha$-SMA and CLIC4 expressions were determined by real-time RT-PCR and Western blotting, when MRC-5 cells were treated with or without antioxidant NAC for $4 \mathrm{~h}$ before addition of CM ${ }^{\text {SKOv } 3}$ and TGF- $\beta 1$ for further 48 h. ${ }^{*} \mathrm{P}<0.05$. (D) ROS mediated up-regulation of CLIC4 expressions in both cytoplasm and nucleus during myofibroblast transdifferentiation. CLIC4 protein expressions in cytoplasm and nucleus were determined by Western blot analysis. MRC-5 cells cultured in serum-free medium served as control for all the experiments.

pro-angiogenesis factors related to myofibroblast differentiation.

Ion transportation through the membrane sets an environment that promotes initiation of cell differentiation programming. The CLIC family, a major class of intracellular chloride channels, comprises so far seven highly homologous members. Serum- or TGF-ß1-stimulated fibroblasts in breast cancer have a gene expression profile of myofibroblasts including a marked up-regulation of CLIC4 $(15,26)$. In vivo, CLIC4 expression markedly increased in stroma of breast, kidney, esophagus and colon cancers (21). We not only found that
CLIC4 expression was up-regulated in epithelial cancer stroma correlated with $\alpha$-SMA expression, but also used an indirect co-culture model to reveal that human primary ovarian fibroblasts and human fibroblast cell line MRC-5 exposed to CM from ovarian cancer cells enhanced CLIC4 and $\alpha$-SMA expressions, which similarly occurred during TGF- $\beta 1-$ induced fibroblast-to-myofibroblast transdifferentiation. We thus conclude that CLIC4 directly participates in stromal fibroblast activation in ovarian cancer.

It has been verified that tumour stromal myofibroblasts favour tumour invasion and metastasis both in experimental 
A



B



C
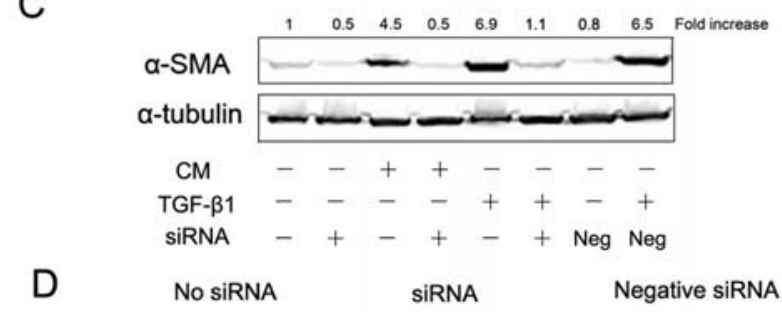

No siRNA

SIRNA

Negative siRNA


$\mathrm{CM}$


TGF- $\beta 1$


Figure 4. CLIC4 is required for myofibroblast transdifferentiation. MRC-5 cells were transfected with specific CLIC4 siRNA and then treated with or without $\mathrm{CM}^{\text {SKOV3 }}$ and TGF-ß1 for $48 \mathrm{~h}$. Mock transfection with transfection reagent alone and negative control siRNA (neg siRNA) served as negative control. (A) The relative level of CLIC4 transcript was assessed by real-time RT-PCR. (B-D) The relative levels of $\alpha$-SMA transcript and protein expression were assessed by real-time RT-PCR, Western blotting and immunofluorescent staining. ${ }^{*} \mathrm{P}<0.05$. Scale bar, $50 \mu \mathrm{m}$.

A



B

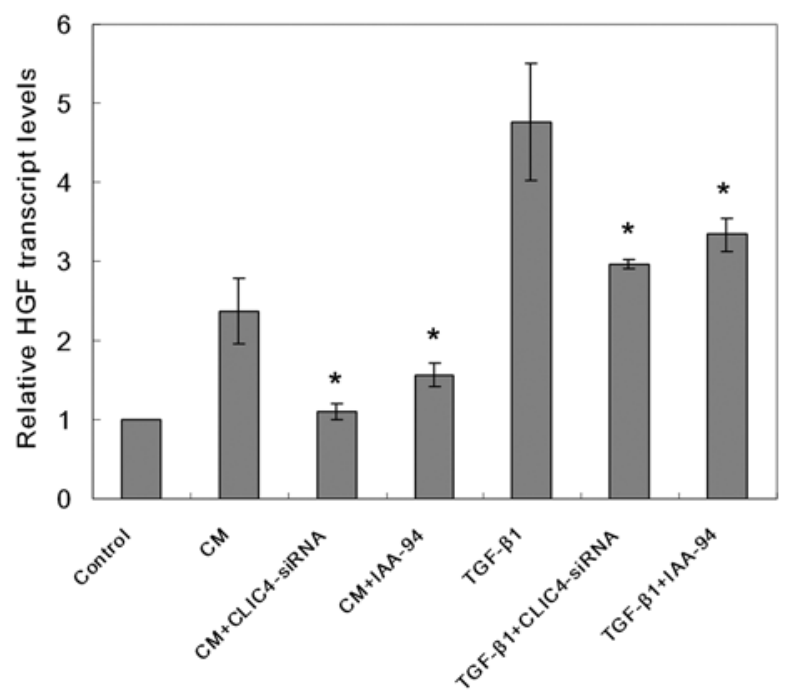

Figure 5. Blocking CLIC4 inhibits the expression of myofibroblast-mediated pro-angiogenic factors. Quantitative PCR was used to analyse transcript levels of VEGF (A) and HGF (B) in MRC-5 cells, when they were treated with or without CLIC4 siRNA or chloride channel inhibitor IAA-94 before addition of $\mathrm{CM}^{\mathrm{SKOV} 3}$ and TGF- $ß 1 .{ }^{*} \mathrm{P}<0.05$.

settings and in clinical specimens $(12,27,28)$. The overexpression of $\alpha$-SMA in tumour stroma is currently used for tumour clinical grading including breast, gastric, colorectal and pancreatic cancer $(27,28)$. CLIC4 co-localized with $\alpha$-SMA in cancer stroma, and the extent of CLIC4 up-regulation correlated with tumour stage and histological grade in kidney, 
colon and breast cancer (21). In this study, we did not observe any significant relationship between CLIC4 expression in stroma and the progression of epithelial ovarian cancer. Further investigations are required to clarify the clinical significance of CLIC4 in ovarian cancer.

ROS can alter gene expression levels, and ROS signalling is involved in TGF-31-induced myofibroblast conversion by regulating the expression of multiple phenotype markers, such as $\alpha$-SMA (10). Our study showed that not only $\alpha$-SMA but also CLIC4 transcription and translation levels were significantly up-regulated following the generation of ROS during TGF- $\beta 1$ induced fibroblast-to-myofibroblast differentiation. This effect could be blocked by pre-treatment with the antioxidant NAC. Furthermore, the crystal structure of CLIC4 is homologous to that of the glutathione S-transferase (GST) superfamily, suggesting its function may depend on the cellular redox state. A cysteine at position 35 preceding the N-terminal transmembrane domain is redox sensitive and is modified by glutathione (16). Thus, ROS may exert an effect on function of CLIC4 through reducing both its expression and activity. It has been reported that CLIC4 can promote cytoskeleton function by interaction with dynamin, tubulin, actin and 14-3-3 proteins (29), and recombinant CLIC4 in fibroblasts reduces cell motility (15). Thus, the relationship between CLIC 4 and $\alpha$-SMA seems to be of consequence in the physiological function. To investigate whether myofibroblast conversion was a consequential outcome of CLIC4 up-regulation, fibroblasts were transfected with specific CLIC4 siRNA prior to TGF- 11 or $\mathrm{CM}^{\text {SKOv3 }}$, which could significantly depress the expression of myofibroblast marker $\alpha$-SMA. Taken together, these data suggest that TGF- $\beta 1$ or $\mathrm{CM}^{\text {SKOV3 }}$ increases cellular ROS, which induces up-regulation of CLIC4, then leads to myofibroblast transdifferentiation.

Recent studies have emphasized the advantage of therapeutic targeting of the tumour stroma, due to the fact that stromal cells reveal genomic stability in contrast to cancer cells, which acquire adaptive mutations during therapy process and these mutations are critical to promote tumour growth. Fibroblasts and myofibroblasts are the major cell types present in various types of human tumour stroma. Therefore, the stromal fibroblast is an attractive target for therapeutic intervention. CLIC4 promotes proliferation and vascular tubulogenesis of endothelial cells in the formation of new blood vessels $(23,30)$. Here, we found that treatment of fibroblasts with the agents blocking CLIC4 had a marked repressive effect on the production of angiogenic factors associated with myofibroblast transdifferentiation, indicating that inhibiting the CLIC4 might have therapeutic potential targeting tumour stroma.

In conclusion, our results not only demonstrate the upregulation of CLIC4 expression during conversion of fibroblasts to myofibroblasts in ovarian cancer, but reveal that tumour-stroma interaction promotes stromal fibroblast activation through ROS-initiated CLIC4 expression. Therefore, inhibiting CLIC4 might be a useful strategy for the prevention of myofibroblast differentiation and myofibroblast-dependent tumour progression. Further investigations of the processes governing myofibroblast transdifferentiation will likely suggest additional approaches to interfere with tumour growth.

\section{Acknowledgements}

This study was supported by the grant from the National Natural Science Foundation of China (No. 30571953) to B.K. and the grant from the National Health and Medical research Council/Cancer council Queensland (No. 401681) to M.W.

\section{References}

1. Jemal A, Siegel R, Ward E, et al: Cancer statistics, 2008. CA Cancer J Clin 58: 71-96, 2008.

2. Ozols RF, Bookman MA, Connolly DC, et al: Focus on epithelial ovarian cancer. Cancer Cell 5: 19-24, 2004.

3. Levi F, Lucchini F, Negri E, Boyle P and La Vecchia C: Cancer mortality in Europe, 1995-1999, and an overview of trends since 1960. Int J Cancer 110: 155-169, 2004.

4. Tlsty TD and Coussens LM: Tumor stroma and regulation of cancer development. Ann Rev Pathol 1: 119-150, 2006.

5. Mueller MM and Fusenig NE: Friends or foes - bipolar effects of the tumour stroma in cancer. Nat Rev 4: 839-849, 2004.

6. Bhowmick NA, Neilson EG and Moses HL: Stromal fibroblasts in cancer initiation and progression. Nature 432: 332-337, 2004.

7. Mahadevan D and von Hoff DD: Tumor-stroma interactions in pancreatic ductal adenocarcinoma. Mol Cancer Ther 6: 1186-1197, 2007.

8. Orimo A, Gupta PB, Sgroi DC, et al: Stromal fibroblasts present in invasive human breast carcinomas promote tumor growth and angiogenesis through elevated SDF-1/CXCL12 secretion. Cell 121: 335-348, 2005.

9. Yang F, Strand DW and Rowley DR: Fibroblast growth factor-2 mediates transforming growth factor-beta action in prostate cancer reactive stroma. Oncogene 27: 450-459, 2008.

10. Cat B, Stuhlmann D, Steinbrenner H, et al: Enhancement of tumor invasion depends on transdifferentiation of skin fibroblasts mediated by reactive oxygen species. J Cell Sci 119 : 2727-2738, 2006.

11. Chaffer CL, Brennan JP, Slavin JL, Blick T, Thompson EW and Williams ED: Mesenchymal-to-epithelial transition facilitates bladder cancer metastasis: role of fibroblast growth factor receptor-2. Cancer Res 66: 11271-11278, 2006.

12. Guo X, Oshima H, Kitmura T, Taketo MM and Oshima M: Stromal fibroblasts activated by tumor cells promote angiogenesis in mouse gastric cancer. J Biol Chem 283: 19864-19871, 2008.

13. Granot D, Addadi Y, Kalchenko V, Harmelin A, KunzSchughart LA and Neeman M: In vivo imaging of the systemic recruitment of fibroblasts to the angiogenic rim of ovarian carcinoma tumors. Cancer Res 67: 9180-9189, 2007.

14. Suh KS, Malik M, Shukla A and Yuspa SH: CLIC4, skin homeostasis and cutaneous cancer: surprising connections. Mol Carcinog 46: 599-604, 2007.

15. Ronnov-Jessen L, Villadsen R, Edwards JC and Petersen OW: Differential expression of a chloride intracellular channel gene, CLIC4, in transforming growth factor-beta1-mediated conversion of fibroblasts to myofibroblasts. Am J Pathol 161: 471-480, 2002.

16. Littler DR, Assaad NN, Harrop SJ, et al: Crystal structure of the soluble form of the redox-regulated chloride ion channel protein CLIC4. FEBS J 272: 4996-5007, 2005.

17. Yang KL, Chang WT, Hung KC, Li EI and Chuang CC: Inhibition of transforming growth factor-beta-induced liver fibrosis by a retinoic acid derivative via the suppression of Col 1A2 promoter activity. Biochem Biophys Res Commun 373: 219-223, 2008.

18. Radisky DC, Levy DD, Littlepage LE, et al: Rac1b and reactive oxygen species mediate MMP-3-induced EMT and genomic instability. Nature 436: 123-127, 2005.

19. Parrott JA, Nilsson E, Mosher R, et al: Stromal-epithelial interactions in the progression of ovarian cancer: influence and source of tumor stromal cells. Mol Cell Endocrinol 175: 29-39, 2001.

20. Vigne JL, Halburnt LL and Skinner MK: Characterization of bovine ovarian surface epithelium and stromal cells: identification of secreted proteins. Biol Reprod 51: 1213-1221, 1994.

21. Suh KS, Crutchley JM, Koochek A, et al: Reciprocal modifications of CLIC4 in tumor epithelium and stroma mark malignant progression of multiple human cancers. Clin Cancer Res 13: 121-131, 2007. 
22. Suh KS, Mutoh M, Mutoh T, et al: CLIC4 mediates and is required for $\mathrm{Ca}^{2+}$-induced keratinocyte differentiation. J Cell Sci 120: 2631-2640, 2007.

23. Bohman S, Matsumoto T, Suh K, et al: Proteomic analysis of vascular endothelial growth factor-induced endothelial cell differentiation reveals a role for chloride intracellular channel 4 (CLIC4) in tubular morphogenesis. J Biol Chem 280: 42397-42404, 2005.

24. Stuhlmann D, Steinbrenner H, Wendlandt B, Mitic D, Sies H and Brenneisen P: Paracrine effect of TGF-beta1 on downregulation of gap junctional intercellular communication between human dermal fibroblasts. Biochem Biophys Res Commun 319: 321-326, 2004

25. Suh KS, Mutoh M, Nagashima K, et al: The organellular chloride channel protein CLIC4/mtCLIC translocates to the nucleus in response to cellular stress and accelerates apoptosis. $\mathbf{J}$ Biol Chem 279: 4632-4641, 2004.

26. Samoszuk M, Tan J and Chorn G: Clonogenic growth of human breast cancer cells co-cultured in direct contact with serumactivated fibroblasts. Breast Cancer Res 7: R274-R283, 2005.
27. Yazhou C, Wenlv S, Weidong Z and Licun W: Clinicopathological significance of stromal myofibroblasts in invasive ductal carcinoma of the breast. Tumour Biol 25: 290-295, 2004.

28. Gulubova MV: Collagen type IV, laminin, alpha-smooth muscle actin (alphaSMA), alpha1 and alpha6 integrins expression in the liver with metastases from malignant gastrointestinal tumours. Clin Exp Metastasis 21: 485-494, 2004.

29. Suginta W, Karoulias N, Aitken A and Ashley RH: Chloride intracellular channel protein CLIC4 (p64H1) binds directly to brain dynamin $\mathrm{I}$ in a complex containing actin, tubulin and 14-3-3 isoforms. Biochem J 359: 55-64, 2001.

30. Ulmasov B, Bruno J, Gordon N, Hartnett ME and Edwards JC: Chloride intracellular channel protein-4 functions in angiogenesis by supporting acidification of vacuoles along the intracellular tubulogenic pathway. Am J Pathol 174: 1084-1096, 2009. 